\title{
Measurement of ternary polymer/solvent equilibrium data by vapor-phase infrared spectroscopy
}

\author{
Yilmaz Yurekli, Sacide Alsoy Altinkaya* \\ Department of Chemical Engineering, Izmir Institute of Technology, Gulbahce Koyu, Urla-Izmir 35437, Turkey
}

\section{A R T I C L E I N F O}

\section{Article history:}

Received 11 April 2008

Received in revised form 3 November 2008

Accepted 4 November 2008

Available online 12 November 2008

\section{Keywords:}

Ternary vapor-liquid equilibrium

Polymer solution

Vapor-phase infrared spectroscopy

Flory-Huggins theory

Entropic and Kannan free volume models

\begin{abstract}
A B S T R A C T
Vapor-liquid equilibrium (VLE) data for binary toluene/PVAC, methanol/PVAC and ternary toluene/methanol/PVAC systems have been measured at $100^{\circ} \mathrm{C}$ by using vapor-phase infrared spectroscopy. Binary data have been compared with literature data measured by different experimental techniques and agreement between our measurements and others was found to be good. The ternary VLE data indicate that the solubility of methanol in PVAC is not influenced by the presence of toluene, while the solubility of toluene is lowered due to presence of methanol. To predict ternary VLE data, the Entropic free volume and Kannan free volume models and the Flory-Huggins theory were used. The predictive abilities of Flory-Huggins theory and the Kannan free volume model are similar and better than that of the Entropic free volume model.
\end{abstract}

(c) 2008 Elsevier B.V. All rights reserved.

\section{Introduction}

Knowledge of vapor liquid equilibrium (VLE) of polymer solutions is essential in the efficient design and optimization of industrial polymer processing unit operations. A few examples include removal of solvents, unreacted monomers and other residues at the end of polymerization, drying of polymer solutions, formulations of paints and coatings and separation of organic mixtures and gases by polymeric membranes.

VLE data for various binary polymer solvent mixtures exist in the literature, however, in some applications, it is necessary to dissolve the polymer in mixed solvents and only very few data are available on VLE of mixed solvents polymer systems. The main reason for the lack of multicomponent equilibrium studies is the difficulty in determining the compositions of both liquid and vapor phases. Combined with a material balance, gas chromatography was the first method used by Katayama and co-workers to measure the ternary VLE data of mixed solvents polymer systems [1,2]. The technique was found to be laborious and difficult to use at elevated temperatures. Therefore, Bonner and co-workers developed a perturbation chromatography technique $[3,4]$ which was also adopted by Glover and his group in measuring ternary VLE data for different polymer solvents systems [3-8]. More recently, the classical gravimetric sorption technique using either quartz spring weigh-

\footnotetext{
* Corresponding author. Tel.: +90 232750 6658; fax: +90 2327506645 .

E-mail address: sacidealsoy@iyte.edu.tr (S.A. Altinkaya).
}

ing $[9,10]$ or a magnetic suspension balance [11] was modified for acquiring sorption data of mixed solvents dissolved in the polymer.

A new analytical technique based on vapor-phase infrared spectroscopy has been developed by Zielinski et al. [12]. The IR technique allows to determine entire sorption isotherms in parallel and facilitates the data acquisition at a much faster rate than conventional methods. In standard gravimetric and volumetric sorption methods, equilibration time to measure each data point on the isotherm can vary from a few hours to 1 day depending on the temperature and sample thickness. In addition, experiments should be conducted in series resulting in a long time to generate the full isotherm. The pressure attainable in most conventional gravimetric and volumetric sorption apparatuses is usually limited to $1 \mathrm{~atm}$. In the case of the IR technique, the upper pressure limit depends only on the quality of the seal made in capping the sample vials.

Zielinski et al. [12] applied the IR technique to measure binary sorption data for the toluene/polyvinyl acetate and methanol/polyvinyl acetate (PVAC) systems at $100^{\circ} \mathrm{C}$, however, they indicated that the method can also be used to measure VLE data in polymeric systems containing multiple solvents if the IR peaks of the solvents can be separated from each other. In this study, we employed the IR technique to measure ternary sorption data for the toluene/methanol/polyvinyl acetate system at $100^{\circ} \mathrm{C}$. The accuracy of our measurements has been confirmed by comparing the toluene/polyvinyl acetate and methanol/polyvinyl acetate data with literature data collected by other experimental techniques. The ternary sorption data were correlated with the Flory-Huggins theory and the Entropic and Kannan free volume models. 


\section{Materials}

Toluene and methanol with the purities of $99.8 \%$ were obtained from Riedel and PVAC (molecular weight: 113,000 g/mol; density: $1.19 \mathrm{~g} / \mathrm{cm}^{3}$ ) was supplied from Aldrich. The $20 \mathrm{ml}$ headspace gas chromatography (GC) vials were purchased from Agilent.

\section{Method}

The basic principles of the IR technique used in this study are given by Zielinski et al. [12]. Briefly, a certain amount of PVAC dried at $100^{\circ} \mathrm{C}$ for $24 \mathrm{~h}$ is put into a GC vial and a known amount of solvent is injected into the vial which is then sealed with an aluminium crimpcap teflon phased septa with a diameter of $20 \mathrm{~mm}$. A teflon disk instead of a conventional septum is used to minimize the experimental error associated with the sorption of solvents into the rubber septum. In order to achieve vapor-liquid equilibria, the sample cell is placed into a temperature-controlled oven. Immediately after removal, it is then inserted into the top of a heater assembly to conduct in situ IR measurement at the specified temperature. A heating assembly made of an aluminium block is selected because of its high thermal conductivity. A schematic illustration of the heater assembly is given in Fig. 1. The inner diameter of the block softly touches the surface of the GC vials so that the heat can be homogenously conducted along the vial. The electrical heater is placed at the bottom of the assembly and it is connected to a PID temperature controller with an accuracy of $\pm 0.5^{\circ} \mathrm{C}$. To minimize the heat loss during analysis, the assembly is covered with a polyurethane insulating material. Two holes at the lateral surfaces of the assembly allow the IR beam to pass through the GC vial. Spectra were obtained at $4 \mathrm{~cm}^{-1}$ resolution using a Digilab Excalibur Series, FTS 3000MX FT-IR instrument equipped with a deuterated triglycine sulfate (DTGS) detector. In order to increase signal to noise ratio, the number of scans was set to 128 for all runs.

To minimize the contribution of the systematic errors caused by sorption of the solvents in the GC vials during analysis of the vapor-phase composition, three important parameters were investigated: position and background of GC vials, sample preparation and temperature of the assembly. While rotating the GC vial does not give any difference in the spectra, variations are observed in the background spectrums between different vials. Therefore, through-

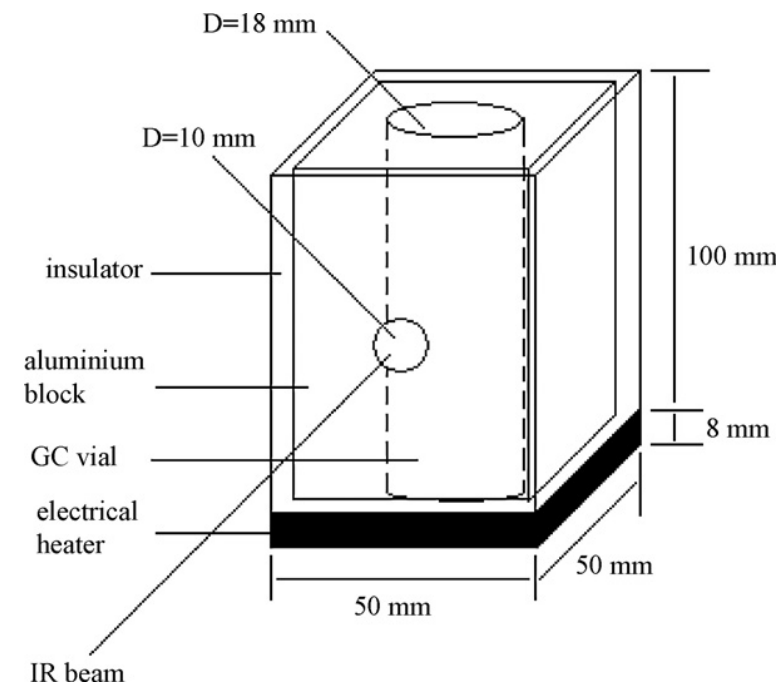

Fig. 1. Schematic representation of the heating assembly used in the FTIR sample chamber.

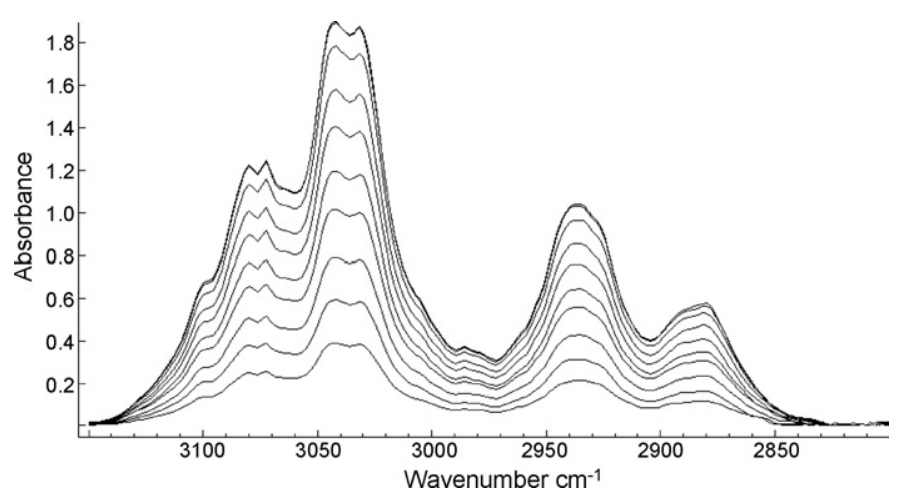

Fig. 2. FT-IR spectra used for the calibration curve of toluene at $100^{\circ} \mathrm{C}$. Spectra correspond to $10-50 \mu \mathrm{l}$ of toluene in increments of $5 \mu \mathrm{l}$.

out the study background spectrums at the specified temperature $\left(100^{\circ} \mathrm{C}\right)$ were obtained for each empty GC vials. All the samples were prepared in a nitrogen glovebox due to sensitivity of the IR technique to water and $\mathrm{CO}_{2}$.

\section{Analysis method}

Quantitative analysis of the IR spectrum were obtained by first constructing a calibration curve. For that purpose, known amounts of methanol and toluene were injected into empty GC vials using $10-100 \mu \mathrm{l}$ syringes. The maximum amount of solvents introduced was $55 \mu \mathrm{l}$ for toluene and $100 \mu \mathrm{l}$ for methanol. These values correspond to saturation volumes at $100^{\circ} \mathrm{C}$ above which condensation of the solvents was observed. The change in absorbance intensities of toluene and methanol with vapor-phase concentrations is illustrated in Figs. 2 and 3, respectively. In these figures, any specific peak of the components can be selected for analyzing the VLE data. Based on the procedure followed by Zielinski et al. [12], we have chosen the $3099.57 \mathrm{~cm}^{-1}$ peak for toluene and the $3680.17 \mathrm{~cm}^{-1}$ peak for methanol. Figs. 4 and 5 show the calibration curves at $100^{\circ} \mathrm{C}$ for toluene and methanol, respectively. By knowing the volume of the vapor phase, the calibration curves were utilized to calculate the mass of the solvents in the vapor phase of the headspace GC vials. While generating the calibration curves, the vapor-phase volume, $V_{\mathrm{g}}$, was taken as the total volume of the vial, $V_{\mathrm{T}}$, which was determined to be $20.4 \mathrm{~cm}^{3}$. During the analysis of polymer/solvents VLE data, it was calculated by subtracting the volume of the polymer from the total volume of the empty vial. Assuming that there is no volume change on mixing, the volume of the polymer, $V_{\mathrm{p}}$, was estimated by summing the volumes of the dry polymer and the solvents

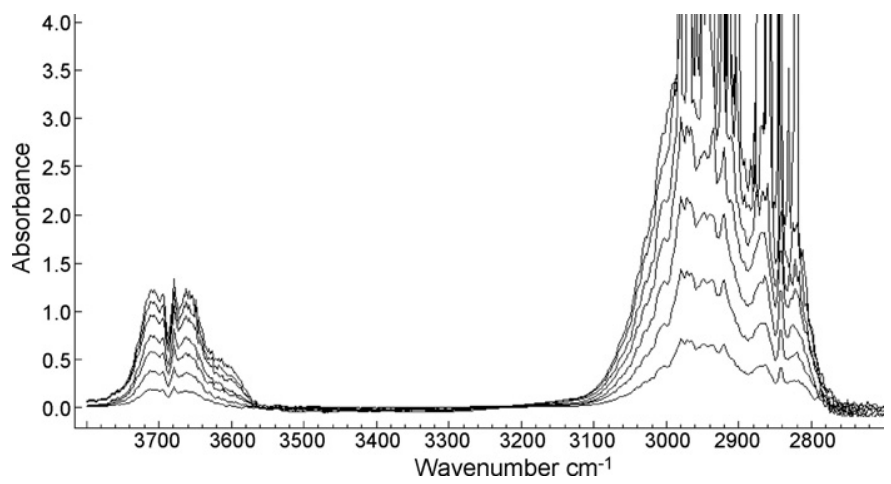

Fig. 3. FT-IR spectra used for the calibration curve of methanol at $100{ }^{\circ} \mathrm{C}$. Spectra correspond to $10-60 \mu \mathrm{l}$ of methanol in increments of $10 \mu \mathrm{l}$. 


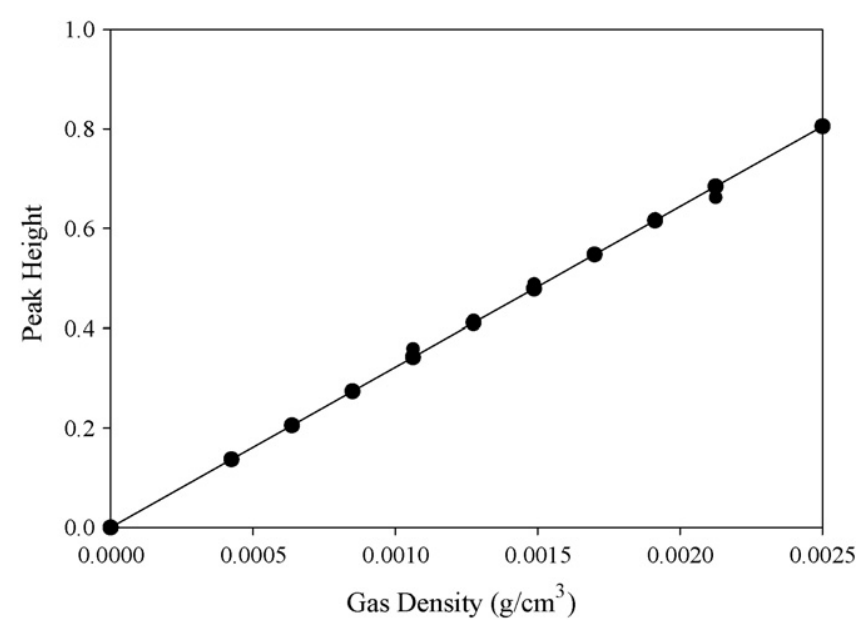

Fig. 4. Calibration curve for the $3099.57 \mathrm{~cm}^{-1}$ toluene IR peak with respect to vaporphase toluene mass density at $100^{\circ} \mathrm{C}$.

absorbed as follows:

$V_{\mathrm{p}}=\frac{m_{\mathrm{pd}}}{\rho_{\mathrm{p}}}+\frac{m_{1 \mathrm{p}}}{\rho_{\mathrm{s} 1}}+\frac{m_{2 \mathrm{p}}}{\rho_{\mathrm{s} 2}}=V_{\mathrm{T}}-V_{\mathrm{g}}$

In Eq. (1), $m_{\mathrm{pd}}$ and $\rho_{\mathrm{p}}$ correspond to the mass and the density of pure polymer, respectively, while $m_{i p}$ represents the mass of solvent $i$ in the polymer phase and $\rho_{s i}$ denotes its pure component density. As shown in Eq. (2), the mass of solvent $i$ in the polymer phase was determined from the difference between the total amount of solvent $i$ introduced into the system, $m_{\mathrm{T} i}=\rho_{i} V_{\mathrm{T} i}$, and the mass of solvent $i$ remained in the vapor phase:

$m_{\text {ip }}=m_{\mathrm{T} i}-\rho_{\mathrm{gi}} V_{\mathrm{g}}$

The vapor-phase concentration of component $i, \rho_{\text {gi }}$, required in Eq. (2) was directly obtained from the calibration curve by detecting the vapor-phase signal intensity $\left(H_{\mathrm{i}}\right)$ during IR measurements:

$\rho_{\mathrm{g} i}=\frac{H_{\mathrm{i}}}{m_{\mathrm{i}}}$

where $m_{\mathrm{i}}$ is the slope of the calibration curve. An expression for the volume of gas phase, $V_{\mathrm{g}}$, is obtained by combining Eqs. (1) and (2):

$V_{\mathrm{g}}=\frac{V_{\mathrm{T}}-\left\{\left(m_{\mathrm{pd}} / \rho_{\mathrm{p}}\right)+\left(m_{\mathrm{T} 1} / \rho_{s 1}\right)+\left(m_{\mathrm{T} 2} / \rho_{s 2}\right)\right\}}{1-\left(\rho_{\mathrm{g} 1} / \rho_{s 1}\right)-\left(\rho_{\mathrm{g} 2} / \rho_{s 2}\right)}$

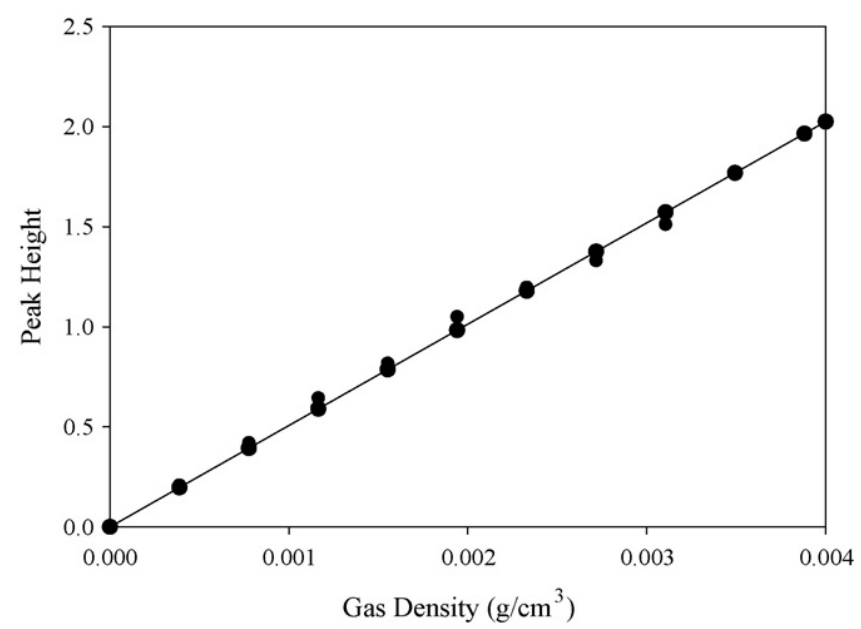

Fig. 5. Calibration curve for the $3680.17 \mathrm{~cm}^{-1}$ methanol IR peak with respect to vapor-phase methanol mass density at $100^{\circ} \mathrm{C}$.
In constructing the sorption isotherms, solvent activities $\left(a_{\mathrm{i}}\right)$ and volume fractions $\left(\phi_{i}\right)$ were calculated as follows:

$a_{\mathrm{i}}=\frac{P_{\mathrm{i}}}{P_{i}^{o}} \quad \phi_{i}=\frac{m_{i \mathrm{p}} / \rho_{s i}}{V_{\mathrm{p}}}$

In Eq. (5), the partial pressure of solvent $i, P_{i}$, was estimated from the ideal gas law:

$P_{i}=\frac{\rho_{\mathrm{g} i} R T}{M_{i}}$

where $M_{i}$ is the molecular weight of solvent $i$, and the vapor pressure of component $i, P_{i}^{o}$, was taken from the literature [13]. The uncertainties in $a_{\mathrm{i}}$ and $\phi_{i}$ caused by uncertainties in the experimental measurements were calculated in Appendix A.

\section{Prediction of sorption data}

To predict the VLE of the toluene/methanol/PVAC system, two purely predictive models (Entropic Free Volume and Kannan Free Volume) and one molecular model (Flory-Huggins) have been used. The free volume models in terms of activity coefficients, $\gamma_{i}$, are of the form [14]:

$\ln \gamma_{i}=\ln \gamma_{i}^{\mathrm{comb}-f v}+\ln \gamma_{i}^{\mathrm{res}}$

where the residual contribution, $\ln \gamma_{i}^{\text {res }}$, is obtained from the UNIFAC residual term [15] and combinatorial/free volume terms are defined below.

\subsection{Entropic free volume model}

$\ln \gamma_{i}^{\mathrm{comb}-f v}=\ln \left(\frac{\phi_{i}^{f v}}{x_{i}}\right)+1-\frac{\phi_{i}^{f v}}{x_{i}}$

where $\phi_{i}^{f v}$ is the free-volume fraction of component $i$

$\phi_{i}^{f v}=\frac{x_{i} v_{i}^{f v}}{\sum_{j} x_{j} v_{j}^{f v}}$

and $x_{i}$ is the mole fraction of component $i$ and $v_{i}^{f v}$ is the free volume of component $i$ which is calculated by subtracting the van der Waals volume of component $i$ from its molar volume.

\subsection{Kannan free volume model}

$\ln \gamma_{i}^{\mathrm{comb}-f v}=\ln \gamma_{i}^{\mathrm{comb}}+\ln \gamma_{i}^{f v}$

The combinatorial contribution, $\ln \gamma_{i}^{\text {comb }}$, in Kannan free volume model is obtained from original UNIFAC expansion, while the free volume term is given as follows:

$\ln \gamma_{i}^{f v}=\ln \left(\frac{\phi_{i}^{f v}}{\phi_{i}^{\mathrm{hc}}}\right)+\left(\frac{\phi_{i}^{\mathrm{hc}}-\phi_{i}^{f v}}{x_{i}}\right)$

The hard-core volume fraction of component $i$ is calculated as

$\phi_{i}^{\mathrm{hc}}=\frac{x_{i} v_{i}^{\mathrm{hc}}}{\sum_{j} x_{j} v_{j}^{\mathrm{hc}}}$

and the hard-core volume of component $i, v_{i}^{\text {hc }}$, is taken to be equal to its van der Waals volume. In the Flory-Huggins theory, the activity 


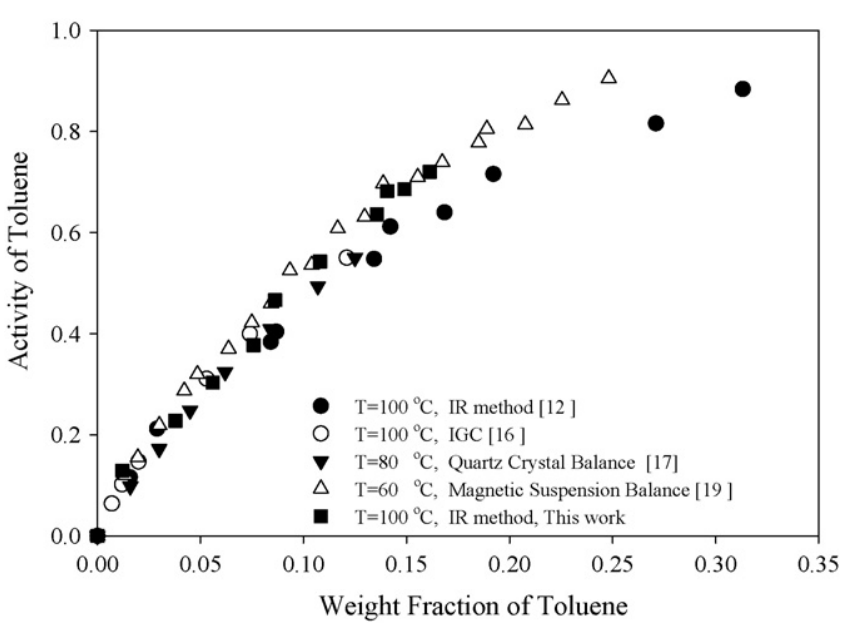

Fig. 6. Comparison of equilibrium sorption data of the toluene/PVAc system measured at $100^{\circ} \mathrm{C}$ by the IR technique and with the literature data.

of the solvents in a ternary system of 2 solvents and a polymer ( 1 , solvent $1 ; 2$, solvent $2 ; 3$, polymer) is given by following equations:

$$
\begin{aligned}
\ln a_{1}= & \ln \phi_{1}+1-\phi_{1}-\frac{V_{1}}{V_{2}} \phi_{2}-\frac{V_{1}}{V_{3}} \phi_{3} \\
& +\left(\chi_{12} \phi_{2}+\chi_{13} \phi_{3}\right)\left(\phi_{2}+\phi_{3}\right)-\chi_{23} \frac{V_{1}}{V_{2}} \phi_{2} \phi_{3}
\end{aligned}
$$

$$
\begin{aligned}
\ln a_{2}= & \ln \phi_{2}+1-\phi_{2}-\frac{V_{2}}{V_{1}} \phi_{1}-\frac{V_{2}}{V_{3}} \phi_{3} \\
& +\left(\chi_{12} \frac{V_{2}}{V_{1}} \phi_{1}+\chi_{23} \phi_{3}\right)\left(\phi_{1}+\phi_{3}\right)-\chi_{13} \frac{V_{2}}{V_{1}} \phi_{1} \phi_{3}
\end{aligned}
$$

In Eqs. (13) and (14), $\phi_{i}$ and $V_{i}$ represent volume fraction and molar volume of component $i$ while $\chi_{i j}$ corresponds to interaction parameter between component $i$ and $j$.

\section{Results and discussion}

To illustrate the accuracy of the IR method, we have first measured the VLE data for toluene/PVAC and methanol/PVAC systems at $100{ }^{\circ} \mathrm{C}$ and compared them with literature data measured by different groups [11,12,16-19]. Methanol sorption data have also been collected in our laboratories (at $60^{\circ} \mathrm{C}$ ) by gravimetric sorp-

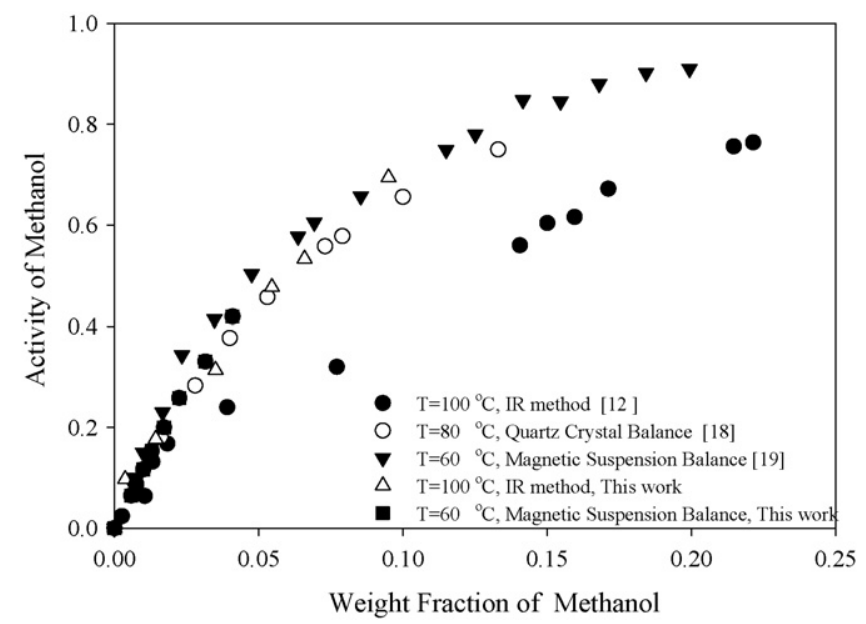

Fig. 7. Comparison of equilibrium sorption data of the methanol/PVAc system measured at $100^{\circ} \mathrm{C}$ by the IR technique and with the literature data.

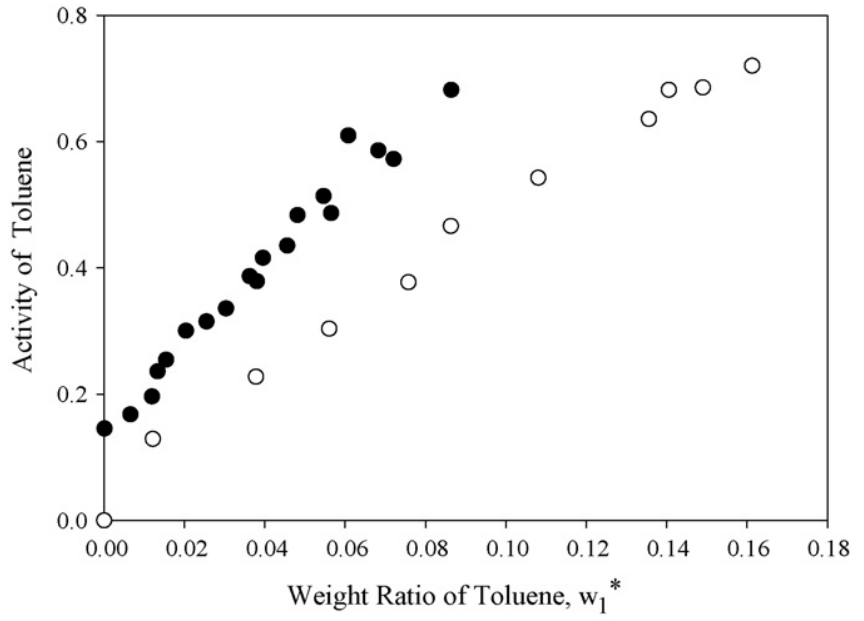

Fig. 8. The change of activity of toluene with respect to its weight ratio in PVAC. Open circles represent data for toluene/PVAC binary system while closed circles represent data for toluene/methanol/PVAC ternary system.

tion using a magnetic suspension balance. The results presented in Figs. 6 and 7 clearly indicate a good agreement between our IR data and those obtained using different experimental techniques. As shown in Fig. 7, methanol solubilities measured by Zielinski et al. [12] using the IR technique are higher than the other data sets and this may be due to difference in molecular weights of the polymers used in different studies. To emphasize the advantage of the IR technique in VLE measurements, it is important to note that most conventional gravimetric sorption devices are limited to $1 \mathrm{~atm}$ of pressure, thus, the maximum activity of methanol achievable at $100^{\circ} \mathrm{C}$ is about 0.29 . On the other hand, the maximum pressure of methanol attained in our measurements was $2.42 \mathrm{~atm}$ which allowed to collect the sorption data up to an activity level of around 0.7. The pressure limitation in the IR technique depends on the quality of the cap seal due to the possibility of solvent leakage.

VLE data for toluene (1)/methanol (2)/PVAC (3) system have been measured at $100^{\circ} \mathrm{C}$. To compare the ternary sorption data with those of the corresponding binary systems, the data are presented in terms of the weight ratio of each solvent absorbed in the polymer: $w_{i}^{*}=m_{s i} /\left(m_{s i}+m_{\mathrm{pd}}\right)$ as a function of the corresponding activities in the vapor phase. As illustrated in Figs. 8 and 9, the solubility of methanol in PVAC is not influenced by the presence of

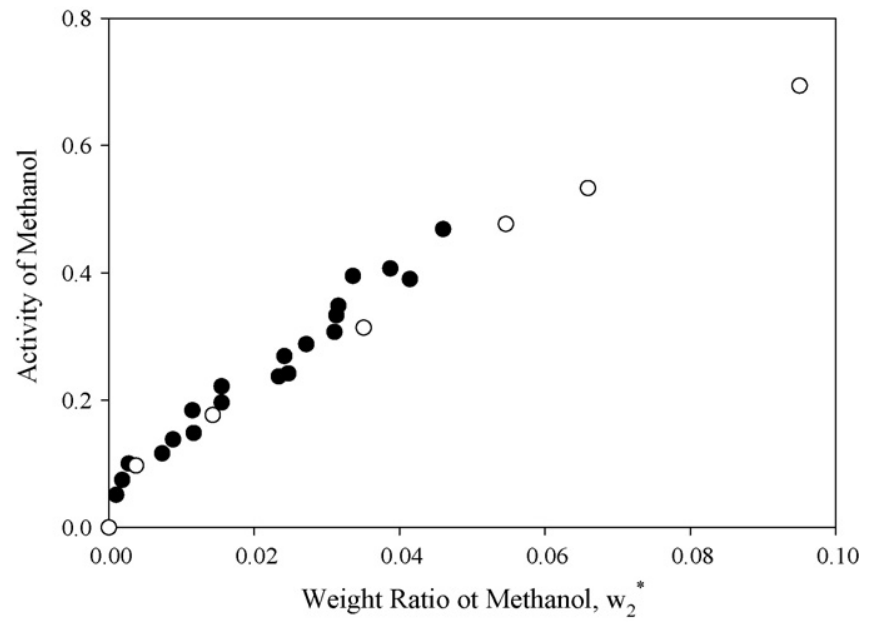

Fig. 9. The change of activity of methanol with respect to its weight ratio in PVAC. Open circles represent data for methanol/PVAC binary system while closed circles represent data for toluene/methanol/PVAC ternary system. 
Table 1

The list of molar volumes and Flory-Huggins interaction parameters for the toluene (1)/methanol (2)/PVAC (3) system.

\begin{tabular}{ll}
\hline Parameter & Value \\
\hline$\chi_{12}$ & $4.39^{\mathrm{a}}$ \\
$\chi_{13}$ & $0.705^{\mathrm{b}}$ \\
$\chi_{23}$ & $1.03^{\mathrm{b}}$ \\
$V_{1}\left(\mathrm{~cm}^{3} / \mathrm{mol}\right)$ & $112.9[13]$ \\
$V_{2}\left(\mathrm{~cm}^{3} / \mathrm{mol}\right)$ & $40.5[13]$ \\
$V_{3}\left(\mathrm{~cm}^{3} / \mathrm{mol}\right)$ & $94,958^{\mathrm{c}}$ \\
\hline \multicolumn{1}{c}{ a } &
\end{tabular}

a Calculated from the solubility parameters: $\chi_{12}=\left(V_{1} / R T\right)\left(\delta_{1}-\delta_{1}\right)^{2}$ $\delta_{1}=8.91\left(\mathrm{cal} / \mathrm{cm}^{3}\right)^{0.5}[13] ; \delta_{2}=14.28\left(\mathrm{cal} / \mathrm{cm}^{3}\right)^{0.5}[13]$.

b Regressed from binary VLE data.

c Calculated from the density and molecular weight of PVAC.

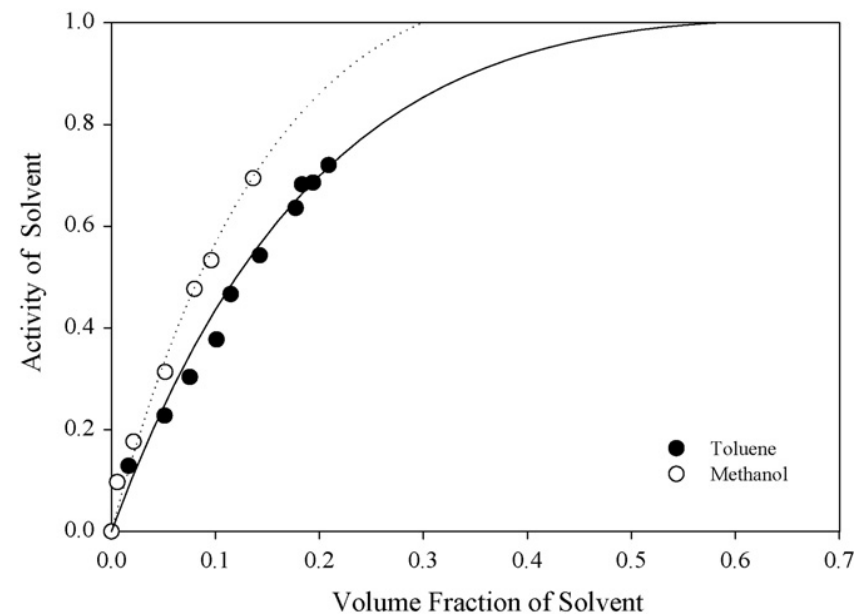

Fig. 10. Equilibrium sorption data for the toluene/PVAC and methanol/PVAC systems measured at $100^{\circ} \mathrm{C}$. The symbols and solid lines represent experimental data and a correlation using the Flory-Huggins model with constant interaction parameter, respectively.

toluene, while the solubility of toluene is lowered due to presence of methanol. The ternary VLE data have been described using the Entropic free volume, Kannan free volume and Flory-Huggins thermodynamic models. The first two models are completely predictive and based on the group contribution principle and activity coeffi-

Table 2

Ternary VLE data for the toluene (1)/methanol (2)/PVAC (3) system measured at $100^{\circ} \mathrm{C}$.

\begin{tabular}{llcl}
\hline$a_{1}$ & $a_{2}$ & \multicolumn{1}{l}{$\phi_{1} \times 100^{\mathrm{a}}$} & $\phi_{2} \times 100$ \\
\hline $0.145 \pm 0.0018$ & $0.075 \pm 0.0029$ & $0.02 \pm 0.0906$ & $0.28 \pm 0.1642^{\mathrm{b}}$ \\
$0.168 \pm 0.0021$ & $0.100 \pm 0.0040$ & $0.90 \pm 0.1168$ & $0.41 \pm 0.2598$ \\
$0.196 \pm 0.0024$ & $0.116 \pm 0.0046$ & $1.61 \pm 0.1264$ & $1.08 \pm 0.3010$ \\
$0.236 \pm 0.0029$ & $0.138 \pm 0.0055$ & $1.80 \pm 0.1268$ & $1.30 \pm 0.3274$ \\
$0.255 \pm 0.0032$ & $0.148 \pm 0.0058$ & $2.07 \pm 0.0975$ & $1.71 \pm 0.2573$ \\
$0.300 \pm 0.0037$ & $0.184 \pm 0.0073$ & $2.73 \pm 0.1376$ & $1.67 \pm 0.4064$ \\
$0.315 \pm 0.0039$ & $0.196 \pm 0.0077$ & $3.39 \pm 0.1283$ & $2.24 \pm 0.3847$ \\
$0.336 \pm 0.0042$ & $0.222 \pm 0.0087$ & $4.03 \pm 0.1303$ & $2.22 \pm 0.4181$ \\
$0.387 \pm 0.0048$ & $0.237 \pm 0.0094$ & $4.75 \pm 0.1734$ & $3.31 \pm 0.5326$ \\
$0.379 \pm 0.0047$ & $0.242 \pm 0.0095$ & $4.97 \pm 0.1296$ & $3.49 \pm 0.4068$ \\
$0.416 \pm 0.0052$ & $0.269 \pm 0.0106$ & $5.17 \pm 0.1355$ & $3.40 \pm 0.4447$ \\
$0.435 \pm 0.0054$ & $0.288 \pm 0.0114$ & $5.92 \pm 0.1438$ & $3.79 \pm 0.4777$ \\
$0.484 \pm 0.0060$ & $0.307 \pm 0.0121$ & $6.21 \pm 0.1623$ & $4.31 \pm 0.5315$ \\
$0.487 \pm 0.0061$ & $0.333 \pm 0.0131$ & $7.27 \pm 0.1667$ & $4.30 \pm 0.5686$ \\
$0.514 \pm 0.0064$ & $0.348 \pm 0.0137$ & $7.03 \pm 0.1537$ & $4.35 \pm 0.5333$ \\
$0.610 \pm 0.0076$ & $0.395 \pm 0.0156$ & $7.79 \pm 0.2309$ & $4.58 \pm 0.7934$ \\
$0.573 \pm 0.0071$ & $0.390 \pm 0.0154$ & $9.10 \pm 0.2031$ & $5.54 \pm 0.6816$ \\
$0.586 \pm 0.0073$ & $0.407 \pm 0.0161$ & $8.66 \pm 0.1757$ & $5.21 \pm 0.6147$ \\
$0.682 \pm 0.0085$ & $0.469 \pm 0.0185$ & $10.80 \pm 0.2593$ & $6.02 \pm 0.8780$
\end{tabular}

a Volume percent.

b The uncertainties were calculated using Eqs. (A.1) through (A.13).
Table 3

Average absolute deviation, $\mathrm{AD}$, between experimental and calculated activities.

\begin{tabular}{ll}
\hline Parameter & $\mathrm{AD}^{\mathrm{a}}$ \\
\hline Entropic FV & 5.21 \\
Kannan FV & 2.68 \\
Flory-Huggins & 2.14
\end{tabular}

$N_{\mathrm{S}}$ : number of solvents. $N_{\mathrm{DP}}$ : number of data points.

$$
\text { a } \mathrm{AD}=\frac{1}{N_{\mathrm{S}}} \sum_{j=1}^{N_{\mathrm{DP}}} \sum_{i=1}^{N_{\mathrm{S}}}\left(a_{j, i}^{\text {calculated }}-a_{j, i}^{\exp }\right) \text {. }
$$

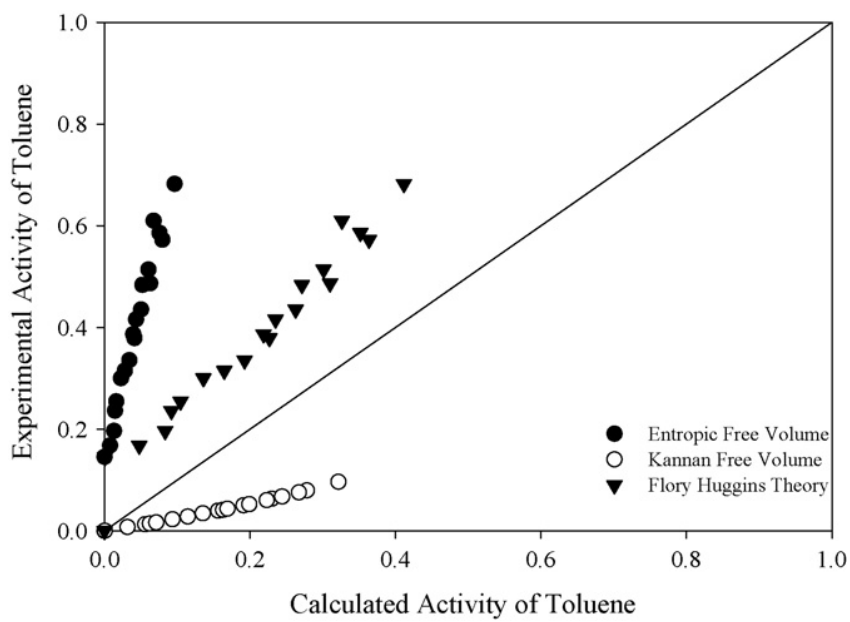

Fig. 11. The activity of toluene predicted by Entropic free volume, Kannan free volume and Flory-Huggins theory against the experimental measurements.

cients are calculated using group and group interaction parameters without any need for parameter regression from binary VLE data. Flory-Huggins theory is a molecular model and requires three interaction parameters, $\chi_{12}, \chi_{13}, \chi_{23}$ and molar volumes for the pure components as input parameters. The interaction parameter between two solvents, $\chi_{12}$, were predicted from solubility parameters [20], while the interaction parameters between solvents and polymer $\left(\chi_{13}, \chi_{23}\right)$ were regressed from binary VLE data by minimizing the following objective function $(\mathrm{OBJ})$ :

$\mathrm{OBJ}=\sum_{i=1}^{N_{\mathrm{DP}}}\left(\ln a_{i}^{\text {calculated }}-\ln a_{i}^{\text {experimental }}\right)^{2}$

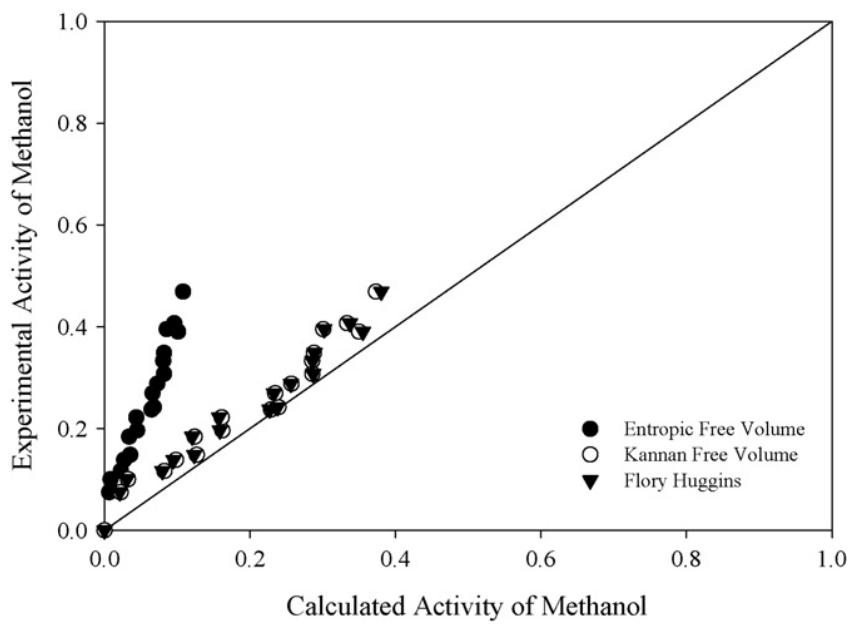

Fig. 12. The activity of methanol predicted by Entropic free volume, Kannan free volume and Flory-Huggins theory against the experimental measurements. 
Regressed/calculated values and other input parameters used in Eqs. (13) and (14) are listed in Table 1. As shown in Fig. 10, the binary sorption data for both methanol and toluene have been correlated well with the Flory-Huggins theory using constant interaction parameters. In Figs. 11 and 12, the activities of solvents calculated by the Entropic free volume, Kannan free volume and Flory-Huggins theory have been plotted against the experimental data. The ternary VLE data and average absolute deviations, AD, between calculated and experimental activities are listed in Tables 2 and 3, respectively. Among the three thermodynamic models tested, the Flory-Huggins theory provides best results, on the other hand, its predictive ability is similar to that of the Kannan free volume model. The Kannan free volume term coupled to UNIFAC in the Kannan free volume model significantly improves its performance compared to Entropic free volume model. It is noted from Figs. 11 and 12 that each model provides better prediction for the activity of methanol.

\section{Conclusion}

In this study, binary and ternary VLE data for the toluene/PVAC, methanol/PVAC and toluene/methanol/PVAC systems have been measured by using a vapor-phase infrared spectroscopy. The accuracy of the experimental technique has been confirmed by good agreement between our binary data and literature data measured by other techniques. The IR technique not only allows to achieve higher pressure than that attainable in conventional gravimetric and volumetric sorption devices but also facilitates VLE data acquisition at a faster rate.

Ternary sorption results reveal that the presence of toluene does not influence the solubility of methanol, on the other hand, the solubility of toluene is lowered due to presence of methanol. Two fundamentally different types of thermodynamic models were used for the prediction of ternary VLE data. On the basis of average absolute deviation between experimental and calculated activities, Flory-Huggins theory provides the best results. The performance of the Kannan free volume model is better than that of Entropic free volume model. To our knowledge, this is the first study illustrating the use of the IR technique to measure VLE data in polymeric systems containing two solvents.

\section{Appendix A}

The uncertainties in the calculated activities and volume fractions of the solvents due to uncertainties in experimentally measured quantities $\left(\delta m_{\mathrm{pd}}, \delta V_{\mathrm{T}}, \delta V_{\mathrm{Ti}}, \delta H_{\mathrm{i}}, \delta m_{\mathrm{i}}, \delta T\right)$ were evaluated by assuming that the measured quantities have a Gaussian distribution about their mean values. According to this assumption, the total error in the activity $\left(\delta a_{i}\right)$ and volume fraction $\left(\delta \phi_{i}\right)$ of solvent $i$ can be obtained by combining the individual contributions in quadrature:

$\delta a_{i}=\sqrt{\left(\frac{\partial a_{i}}{\partial H_{i}}\right)^{2} \delta H_{i}^{2}+\left(\frac{\partial a_{i}}{\partial m_{i}}\right)^{2} \delta m_{i}^{2}+\left(\frac{\partial a_{i}}{\partial T}\right)^{2} \delta T^{2}}$

$$
\begin{aligned}
& A=\left(\frac{\partial \phi_{i}}{\partial m_{\mathrm{pd}}}\right)=\left(\frac{\partial \phi_{i}}{\partial m_{\mathrm{pd}}}\right)+\sum_{j=1}^{2}\left(\frac{\partial \phi_{i}}{\partial m_{\mathrm{jp}}}\right)\left(\frac{\partial m_{\mathrm{jp}}}{\partial V_{\mathrm{g}}}\right)\left(\frac{\partial V_{\mathrm{g}}}{\partial m_{\mathrm{pd}}}\right) \\
& B=\left(\frac{\partial \phi_{i}}{\partial V_{\mathrm{T}}}\right)=\sum_{j=1}^{2}\left(\frac{\partial \phi_{i}}{\partial m_{\mathrm{jp}}}\right)\left(\frac{\partial m_{\mathrm{jp}}}{\partial V_{\mathrm{g}}}\right)\left(\frac{\partial V_{\mathrm{g}}}{\partial V_{\mathrm{T}}}\right) \\
& C=\left(\frac{\partial \phi_{i}}{\partial V_{\mathrm{T} 1}}\right)=\left(\frac{\partial \phi_{i}}{\partial m_{1 \mathrm{p}}}\right)\left(\frac{\partial m_{1 \mathrm{p}}}{\partial m_{\mathrm{T} 1}}\right)\left(\frac{\partial m_{\mathrm{T} 1}}{\partial V_{\mathrm{T} 1}}\right) \\
& +\sum_{j=1}^{2}\left(\frac{\partial \phi_{i}}{\partial m_{\mathrm{jp}}}\right)\left(\frac{\partial m_{\mathrm{jp}}}{\partial V_{\mathrm{g}}}\right)\left(\frac{\partial V_{\mathrm{g}}}{\partial m_{\mathrm{T} 1}}\right)\left(\frac{\partial m_{\mathrm{T} 1}}{\partial V_{\mathrm{T} 1}}\right) \\
& D=\left(\frac{\partial \phi_{i}}{\partial V_{\mathrm{T} 2}}\right)=\left(\frac{\partial \phi_{i}}{\partial m_{2 \mathrm{p}}}\right)\left(\frac{\partial m_{2 \mathrm{p}}}{\partial m_{\mathrm{T} 2}}\right)\left(\frac{\partial m_{\mathrm{T} 2}}{\partial V_{\mathrm{T} 2}}\right) \\
& +\sum_{j=1}^{2}\left(\frac{\partial \phi_{i}}{\partial m_{\mathrm{jp}}}\right)\left(\frac{\partial m_{\mathrm{jp}}}{\partial V_{\mathrm{g}}}\right)\left(\frac{\partial V_{\mathrm{g}}}{\partial m_{\mathrm{T} 2}}\right)\left(\frac{\partial m_{\mathrm{T} 2}}{\partial V_{\mathrm{T} 2}}\right) \\
& E=\left(\frac{\partial \phi_{i}}{\partial H_{1}}\right)=\left(\frac{\partial \phi_{i}}{\partial m_{1 \mathrm{p}}}\right)\left(\frac{\partial m_{1 \mathrm{p}}}{\partial \rho_{\mathrm{g} 1}}\right)\left(\frac{\partial \rho_{\mathrm{g} 1}}{\partial H_{1}}\right) \\
& +\sum_{j=1}^{2}\left(\frac{\partial \phi_{i}}{\partial m_{\mathrm{jp}}}\right)\left(\frac{\partial m_{\mathrm{jp}}}{\partial V_{\mathrm{g}}}\right)\left(\frac{\partial V_{\mathrm{g}}}{\partial \rho_{\mathrm{g} 1}}\right)\left(\frac{\partial \rho_{\mathrm{g} 1}}{\partial H_{1}}\right) \\
& F=\left(\frac{\partial \phi_{i}}{\partial H_{2}}\right)=\left(\frac{\partial \phi_{i}}{\partial m_{2 \mathrm{p}}}\right)\left(\frac{\partial m_{2 \mathrm{p}}}{\partial \rho_{\mathrm{g} 2}}\right)\left(\frac{\partial \rho_{\mathrm{g} 2}}{\partial H_{2}}\right) \\
& +\sum_{j=1}^{2}\left(\frac{\partial \phi_{i}}{\partial m_{\mathrm{jp}}}\right)\left(\frac{\partial m_{\mathrm{jp}}}{\partial V_{\mathrm{g}}}\right)\left(\frac{\partial V_{\mathrm{g}}}{\partial \rho_{\mathrm{g} 2}}\right)\left(\frac{\partial \rho_{\mathrm{g} 2}}{\partial H_{2}}\right) \\
& G=\left(\frac{\partial \phi_{i}}{\partial m_{1}}\right)=\left(\frac{\partial \phi_{i}}{\partial m_{1 \mathrm{p}}}\right)\left(\frac{\partial m_{1 \mathrm{p}}}{\partial \rho_{\mathrm{g} 1}}\right)\left(\frac{\partial \rho_{\mathrm{g} 1}}{\partial m_{1}}\right) \\
& +\sum_{j=1}^{2}\left(\frac{\partial \phi_{i}}{\partial m_{\mathrm{jp}}}\right)\left(\frac{\partial m_{\mathrm{jp}}}{\partial V_{\mathrm{g}}}\right)\left(\frac{\partial V_{\mathrm{g}}}{\partial \rho_{\mathrm{g} 1}}\right)\left(\frac{\partial \rho_{\mathrm{g} 1}}{\partial m_{1}}\right) \\
& F=\left(\frac{\partial \phi_{i}}{\partial m_{2}}\right)=\left(\frac{\partial \phi_{i}}{\partial m_{2 \mathrm{p}}}\right)\left(\frac{\partial m_{2 \mathrm{p}}}{\partial \rho_{\mathrm{g} 2}}\right)\left(\frac{\partial \rho_{\mathrm{g} 2}}{\partial m_{2}}\right) \\
& +\sum_{j=1}^{2}\left(\frac{\partial \phi_{i}}{\partial m_{\mathrm{jp}}}\right)\left(\frac{\partial m_{\mathrm{jp}}}{\partial V_{\mathrm{g}}}\right)\left(\frac{\partial V_{\mathrm{g}}}{\partial \rho_{\mathrm{g} 2}}\right)\left(\frac{\partial \rho_{\mathrm{g} 2}}{\partial m_{2}}\right)
\end{aligned}
$$

The derivatives required in Eqs. (A.3)-(A.13) were calculated using Eqs. (1)-(6).

$\delta \phi_{i}=\sqrt{A^{2} \delta m_{\mathrm{pd}}^{2}+B^{2} \delta V_{\mathrm{T}}^{2}+C^{2} \delta V_{\mathrm{T} 1}^{2}+D^{2} \delta V_{\mathrm{T} 2}^{2}+E^{2} \delta H_{1}^{2}+F^{2} \delta H_{2}^{2}+G^{2} \delta m_{1}^{2}+H^{2} \delta m_{2}^{2}}$

where the partial derivatives appearing in Eqs. (A.1) and (A.2) are defined as follows:

$$
\begin{aligned}
& \left(\frac{\partial a_{i}}{\partial H_{i}}\right)=\left(\frac{\partial a_{i}}{\partial P_{i}}\right)\left(\frac{\partial P_{i}}{\partial \rho_{g i}}\right)\left(\frac{\partial \rho_{g i}}{\partial H_{i}}\right) \\
& \left(\frac{\partial a_{i}}{\partial m_{i}}\right)=\left(\frac{\partial a_{i}}{\partial P_{i}}\right)\left(\frac{\partial P_{i}}{\partial \rho_{g i}}\right)\left(\frac{\partial \rho_{g i}}{\partial m_{i}}\right) \\
& \left(\frac{\partial a_{i}}{\partial T}\right)=\left(\frac{\partial a_{i}}{\partial P_{i}}\right)\left(\frac{\partial P_{i}}{\partial T}\right)
\end{aligned}
$$

\section{References}

[1] T. Katayama, K. Matsumura, Y. Urahama, Kagaku Kogaku 35 (1971) 1012-1017.

[2] K. Matsumura, T. Katayama, Kagaku Kogaku 38 (1974) 388-392.

[3] D.C. Bonner, N.F. Brockmeier, Ind. Eng. Chem., Process Des. Dev. 16 (1977) 180-186.

[4] S. Dinçer, D.C. Bonner, R.A. Elefritz, Ind. Eng. Chem. Fundam. 18 (1979) 54-59.

[5] W.A. Ruff, C.J. Glover, A.T. Watson, AIChE J. 32 (1986) 1948-1953.

[6] W.A. Ruff, C.J. Glover, A.T. Watson, W.R. Lau, J.C. Holste, AIChE J. 32 (1986) 1954-1962.

[7] T.K. Tsotsis, C. Turchi, C.J. Glover, Macromolecules 20 (1987) 2445-2452. 
[8] L.L. Joffrion, C.J. Glover, Macromolecules 19 (1986) 1710-1718.

[9] H. Liu, Y. Huang, K. Wang, Y. Hu, Fluid Phase Equilibr. 194-197 (2002) 1067-1075.

[10] J.O. Tanbonliong, J.M. Prausnitz, Polymer 38 (1997) 5775-5783.

[11] W. Schabel, P. Scharfer, M. Kind, I. Mamaliga, Chem. Eng. Sci. 62 (2007) 2254-2266.

[12] J.M. Zielinski, B.T. Carvill, S.A. Gardner, M.F. Kimak, R. Horvath, J.E. Rovira, Ind. Eng. Chem. Res. 40 (2001) 2990-2994.

[13] T.E. Daubert, R.P. Danner, Physical and Thermodynamic Properties of Pure Compounds: Data Compilation, Taylor \& Francis, New York, 1994.

[14] H.R. Radfarnia, G.M. Kontogeorgis, C. Ghotbi, V. Taghikhani, Fluid Phase Equilibr. 257 (2007) 63-69.
[15] Aa. Fredenslund, R.L. Jones, J.M. Prausnitz, AIChE J. 21 (1975) 1086-1099.

[16] F. Tihminlioglu, R.P. Danner, N. Lützow, J.L. Duda, J. Polym. Sci. Part B: Polym. Phys. 38 (2000) 2429-2435.

[17] G. Wibawa, M. Takahashi, Y. Sato, S. Takishima, H. Masuoka, J. Chem. Eng. Data 47 (2002) 518-524.

[18] G. Wibawa, R. Hatano, Y. Sato, S. Takishima, H. Masuoka, J. Chem. Eng. Data 47 (2002) 1022-1029.

[19] I. Mamaliga, W. Schabel, M. Kind, Chem. Eng. Process. 43 (2004) 753-763.

[20] T. Lindvig, M.L. Michelsen, G.M. Kontogeorgis, Fluid Phase Equilibr. 203 (2002) 247-260. 